\title{
Pelvic panniculitis mimicking abscess in a patient with systemic lupus erythematosus
}

Singapore Med J 2021; 62(2): 104-105 https://doi.org/10.11622/smedj.2021018

Dear Sir,

Panniculitis is defined as inflammation of adipose tissue and usually occurs subcutaneously. ${ }^{(1)}$ To date, pelvic panniculitis has not been described in the literature. We herein report a case with an initial working diagnosis of a perirectal abscess that was intraoperatively and histologically proven to be panniculitis. With careful inspection of morphological and functional imaging features on magnetic resonance (MR) imaging, it may be possible to diagnose panniculitis preoperatively, potentially avoiding unnecessary surgery. This case highlights panniculitis as a rare differential diagnosis and MR imaging as a promising non-invasive tool in making the diagnosis.

A 43-year-old Chinese woman was diagnosed with systemic lupus erythematosus (SLE) at the age of 30 years. She was treated with low-dose steroids and hydroxychloroquine (HCQ) over the years. Her SLE remained clinically quiescent despite the presence of active serological markers. There was no renal, gastrointestinal or central nervous system involvement. For the present admission, she presented with right-sided buttock pain and fever of four days' duration. There was no rectal bleeding, cutaneous discharge or association with bowel opening. There was no preceding trauma. Physical examination revealed a temperature of $38.2^{\circ} \mathrm{C}$ and tenderness at the anal verge and 8-9 o'clock position. Inflammatory markers including white cell count and C-reactive protein level were elevated at $14.57 \times 10^{9} / \mathrm{L}$ and $26 \mathrm{mg} / \mathrm{L}$, respectively.

MR imaging of the pelvis followed by examination under anaesthesia (EUA) was performed, in view of the strong clinical suspicion of a perirectal abscess (Fig. 1). Intraoperatively, there was no drainable collection or pus. The right ischiorectal fossa fat pad was indurated and blunt dissection showed loose areolar and inflamed fatty tissue. Histology revealed non-specific inflammatory and reactive changes. The culture grew scanty Escherichia coli and Staphylococcus lugdunensis. Repeat MR imaging (Fig. 2) and EUA one week later showed similar findings of inflammation with no collection identified. The patient's symptoms improved after a week of intravenous clindamycin, five days of metronidazole and two weeks of levofloxacin, prescribed according to culture sensitivity results. Colonoscopy was normal. She was discharged after a three-week hospital stay. Her oral prednisolone was titrated, and she was discharged with the same dosage of HCQ $200 \mathrm{mg}$ and oral prednisolone. She remained well subsequently.

Panniculitis is histologically divided into two main types: mostly septal or mostly lobular, ${ }^{(2,3)}$ although the two types have some overlapping features. ${ }^{(2,4)}$ Each of them is further classified into panniculitis with or without vasculitis. Lupus erythematosus panniculitis, also known as lupus profundus, is an example of a mostly lobular type of panniculitis without vasculitis. ${ }^{(4)}$ Panniculitis comprises only $1 \%-3 \%$ of SLE cutaneous manifestations. ${ }^{(4)}$ Another entity of panniculitis occurring in the abdomen is mesenteric panniculitis. SLE is one of the extremely rare causes of mesenteric panniculitis. From our literature search, there were only two reported cases of SLE presenting as mesenteric panniculitis. ${ }^{(5)}$

Pelvic vasculitis in patients with SLE has rarely been described. Traisak et al reported that pelvic vasculitis involving the uterus, cervix and fallopian tubes was histologically diagnosed in a patient who presented with a large pelvic haematoma. ${ }^{(6)}$ Only a total of three case reports, including two cases of ovarian ${ }^{(7,8)}$ and one case of uterine ${ }^{(9)}$ vasculitis, have been reported among SLE patients. From the English literature search of PubMed, MEDLINE®, Medknow Publications and Google Scholar, to our knowledge, no similar case of panniculitis involving the ischiorectal or ischioanal fat has been reported. It is a diagnostic challenge because the clinical manifestations overlap with those of an abscess, which is far more common.

Our patient was treated for lupus panniculitis and concurrent acute infection with immunosuppressants and antibiotics. We were unable to ascertain if the panniculitis was secondary to lupus, infection, or both, as the patient had improved with the treatment combination.
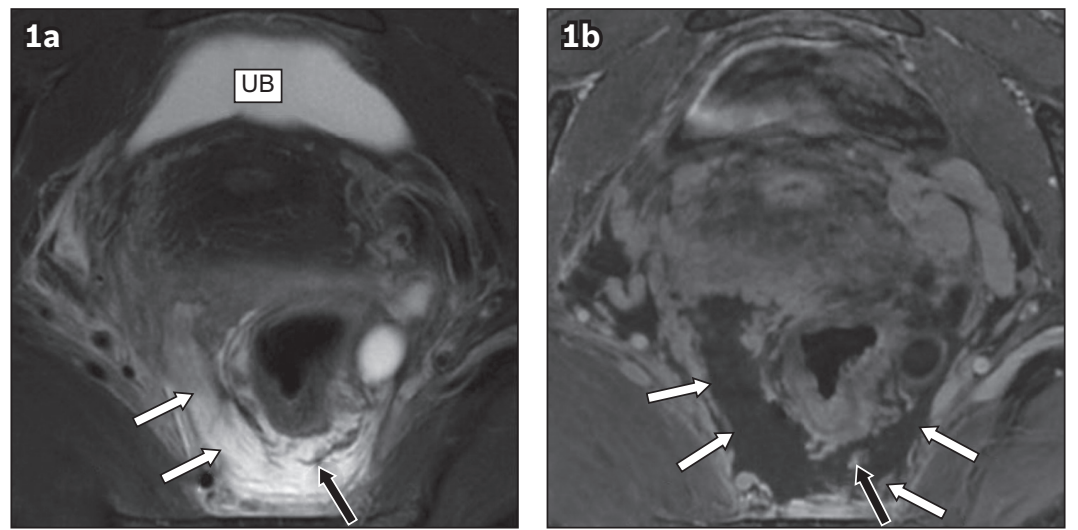

Fig. 1 (a) Preoperative axial T2-W fat-saturated MR image shows an area of T2-W hyperintense change around the rectum (white arrows), with a signal intensity approaching that of fluid in the urinary bladder (UB). (b) Preoperative axial T1-W LAVA-Flex post-gadolinium enhanced MR image shows irregular peripheral enhancement (white arrows) around the T2-W hyperintense change, resembling a large horseshoe perirectal abscess with irregular wall enhancement. On careful inspection of the T2-W images, there were vessels (black arrow in a) traversing the inflamed area, which is atypical for an abscess/collection. These vessels showed expected opacification post gadolinium (black arrow in b). 

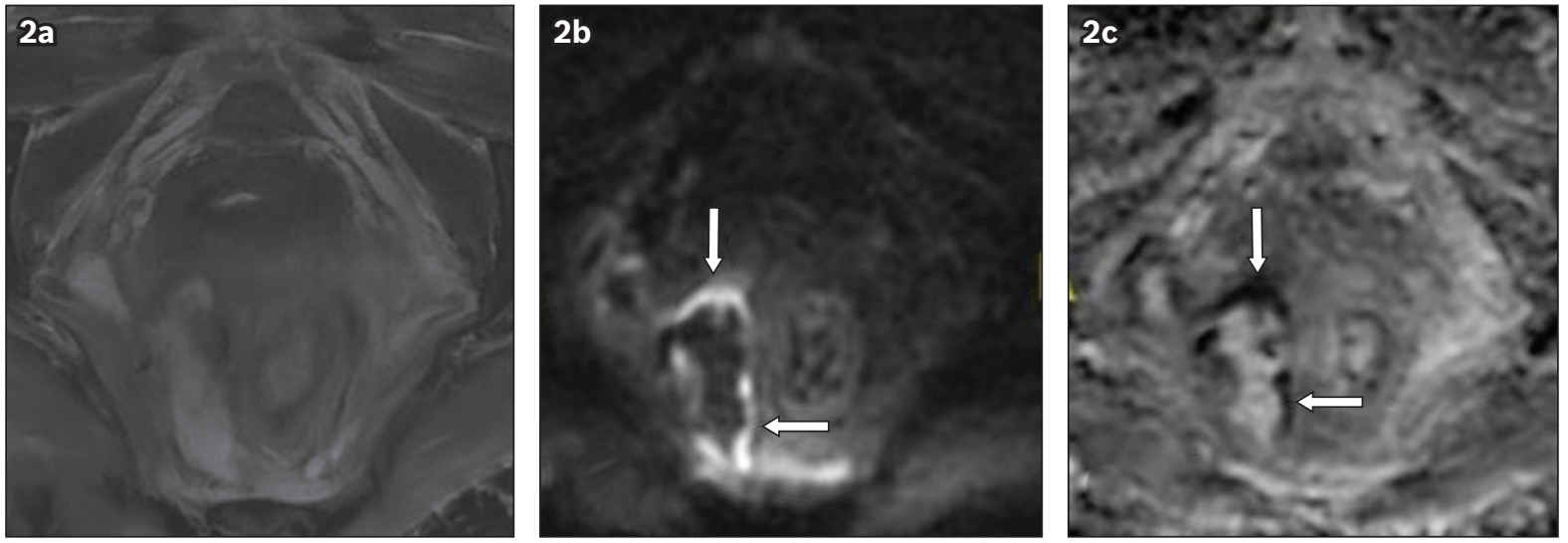

Fig. 2 (a) Axial T2-W fat-saturated sequence, (b) diffusion-weighted imaging (DWI) sequence and (c) calculated apparent diffusion coefficient (ADC) sequence MR images one week postoperatively show only a thin rim of restricted diffusion at the periphery of the T2-W hyperintense change (arrows). DWI and ADC sequences were not part of the initial protocol that was ordered preoperatively and were only obtained on follow-up in view of the operative findings.

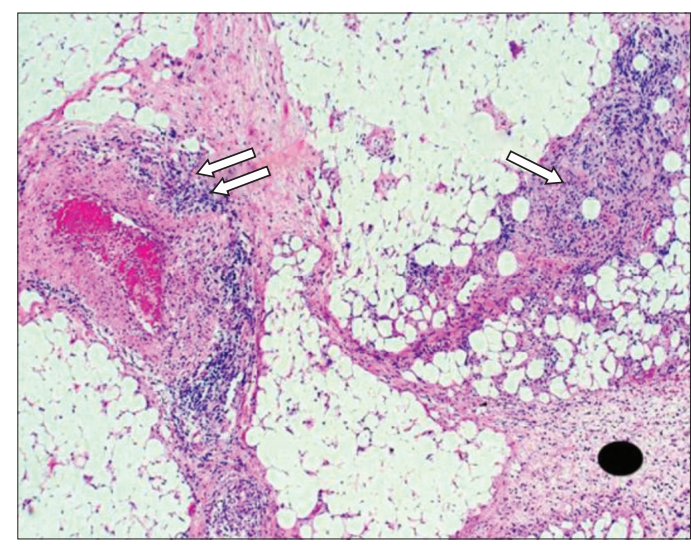

Fig. 3 Intraoperative photomicrograph of the ischiorectal fat shows fibrous expanded septae with septal and lobular inflammatory infiltrates (arrows) with no evidence of vasculitis (Haemotoxylin \& eosin, $\times 40$ ).
MR imaging remains the primary, non-invasive imaging tool we would recommend in patients with a suspected perianal abscess or fistula. It mainly assists in diagnosing and evaluating the extent of disease for preoperative planning. In our patient, the MR imaging features of intense inflammatory change and peripheral enhancement resulted in the panniculitis being mistaken for a large horseshoe-shaped abscess in perirectal and perianal fat. In differentiating an abscess from panniculitis, we have found that it is useful to carefully look for vessels that traverse the lesion, as this implies the preservation of stroma and connective tissue around the anorectum. A fluid collection would displace vessels rather than allow the vessel to traverse through it. Secondly, restriction diffusion is usually seen in an abscess centrally because of constrained movement of water molecules in pus and necrotic material. ${ }^{(10)}$ The absence of restricted diffusion should alert the

clinician to a non-abscess aetiology. The above features would favour panniculitis over an abscess and defer operative drainage for patients in this group.

In summary, pelvic panniculitis is a rare mimicker of an abscess and has not been described in the literature. We have found some MR imaging features that could be discriminatory, namely the preservation of vessels coursing through the affected area and the absence of restricted diffusion within the lesion.

Yours sincerely,

Yin Eie $\underline{T e o}^{1}$, Bengt Fredrik Petersson ${ }^{2}$, Ridzuan $\underline{\text { Farouk }}^{3}$, Wynne Yuru Chua ${ }^{1}$

${ }^{1}$ Department of Diagnostic Imaging, ${ }^{2}$ Department of Pathology, ${ }^{3}$ Department of Surgery, Division of Colorectal Surgery, National University Hospital, Singapore. yineie@hotmail.com

\section{References}

1. Diaz Cascajo C, Borghi S, Weyers W. Panniculitis: definition of terms and diagnostic strategy. Am J Dermatopathol 2000; 22:530-49.

2. Requena L, Yus ES. Panniculitis. Part I. Mostly septal panniculitis. J Am Acad Dermatol 2001; 45:163-83; quiz 184-6.

3. Requena L, Sánchez Yus E. Panniculitis. Part II. Mostly lobular panniculitis. J Am Acad Dermatol 2001; 45:325-61; quiz 362-4.

4. Gupta P, Saikia U, Arora S, De D, Radotra B. Panniculitis: a dermatopathologist's perspective and approach to diagnosis. Indian J Dermatopathol Diagn Dermatol $2016 ; 3: 29-41$.

5. D Pahuja, M Eustace, P Chadha. Mesenteric panniculitis: an unusual initial presentation of systemic lupus erythematosus (SLE). Internet J Rheumatol 2005; 2:8.

6. Traisak P, Basnyat S, Eid H, et al. A unique case of systemic lupus erythematosus pelvic vasculitis. Case Rep Rheumatol 2016; $2016: 6347901$.

7. Massasso D, Cheruvu C, Joshua F, Yong J, Gotis-Graham IG. Ovarian vasculitis in an adult with fatal systemic lupus erythematosus. Lupus 2009; 18:364-67.

8. Meyers KE, Pfieffer S, Lu T, Kaplan BS. Genitourinary complications of systemic lupus erythematosus. Pediatr Nephrol 2000; 14:416-21.

9. Feriozzi S, Muda AO, Amini M, Faraggiana T, Ancarani E. Systemic lupus erythematosus with membranous glomerulonephritis and uterine vasculitis. Am J Kidney Dis $1997 ; 29: 277-9$.

10. Unal O, Koparan HI, Avcu S, Kalender AM, Kisli E. The diagnostic value of diffusion-weighted magnetic resonance imaging in soft tissue abscesses. Eur J Radiol 2011; 77:490-4. 